\title{
Resistive MHD reconstruction of two-dimensional coherent structures in space
}

\author{
W.-L. Teh ${ }^{1}$, B. U. Ö. Sonnerup ${ }^{2}$, J. Birn ${ }^{3}$, and R. E. Denton ${ }^{2}$ \\ ${ }^{1}$ Laboratory for Atmospheric and Space Physics, Boulder, CO, USA \\ ${ }^{2}$ Dartmouth College, Hanover, NH, USA \\ ${ }^{3}$ Los Alamos National Laboratory, Los Alamos, NM, USA
}

Received: 27 May 2010 - Revised: 21 October 2010 - Accepted: 10 November 2010 - Published: 24 November 2010

\begin{abstract}
We present a reconstruction technique to solve the steady resistive MHD equations in two dimensions with initial inputs of field and plasma data from a single spacecraft as it passes through a coherent structure in space. At least two components of directly measured electric fields (the spacecraft spin-plane components) are required for the reconstruction, to produce two-dimensional (2-D) field and plasma maps of the cross section of the structure. For convenience, the resistivity tensor $\boldsymbol{\eta}$ is assumed diagonal in the reconstruction coordinates, which allows its values to be estimated from Ohm's law, $\boldsymbol{E}+\boldsymbol{v} \times \boldsymbol{B}=\boldsymbol{\eta} \cdot \boldsymbol{j}$. In the present paper, all three components of the electric field are used. We benchmark our numerical code by use of an exact, axisymmetric solution of the resistive MHD equations and then apply it to synthetic data from a 3-D, resistive, MHD numerical simulation of reconnection in the geomagnetic tail, in a phase of the event where time dependence and deviations from 2-D are both weak. The resistivity used in the simulation is time-independent and localized around the reconnection site in an ellipsoidal region. For the magnetic field, plasma density, and pressure, we find very good agreement between the reconstruction results and the simulation, but the electric field and plasma velocity are not predicted with the same high accuracy.
\end{abstract}

Keywords. Magnetospheric physics (Magnetopause, cusp, and boundary layers) - Space plasma physics (Magnetic reconnection; Numerical simulation studies)

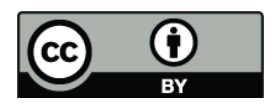

Correspondence to: W.-L. Teh (waileong.teh@gmail.com)

\section{Introduction}

Over the past few years, reconstruction of two-dimensional (2-D), time-independent field and plasma structures from data, taken by a single spacecraft as it passes through the structures, has been frequently used for the analysis and interpretation of space data. The pioneering reconstruction method was developed for cases governed by the classical Grad-Shafranov (GS) equation, in which the plasma flows are assumed negligible (Sonnerup and Guo, 1996; Hau and Sonnerup, 1999). GS reconstruction of this type has been successfully applied in various studies, e.g., of magnetopause structures (Hau and Sonnerup, 1999; Hu and Sonnerup, 2000, 2003; Hasegawa et al., 2004, 2005; Teh and Hau, 2004, 2007), including flux transfer events (FTEs) (Sonnerup et al., 2004; Hasegawa et al., 2006; Lui et al., 2008), and also of flux ropes in the geomagnetic tail (Hasegawa et al., 2007a) and magnetic flux ropes and cloud structures in the solar wind (Hu and Sonnerup, 2001, 2002). Sonnerup et al. (2006) extended the GS reconstruction method to the case where substantial field-aligned flow is present (see Teh et al., 2007), and also to the case of streamline reconstruction in flow perpendicular to a unidirectional magnetic field (see Hasegawa et al., 2007b; Eriksson et al., 2009).

Recently, Sonnerup and Teh (2008) showed that reconstruction can be done directly from the steady, 2-D, ideal MHD equations. In ideal MHD reconstruction, both magnetic field and velocity field maps can be generated, whereas the GS-based reconstruction produces either a magnetic field or a velocity field map. The ideal-MHD reconstruction capability has thus removed the severe restrictions imposed by the GS equation. It has now been applied to reconnection events at the magnetopause (Teh and Sonnerup, 2008) and in

Published by Copernicus Publications on behalf of the European Geosciences Union. 
the solar wind (Teh et al., 2009), and also to FTEs associated with Kelvin-Helmholtz waves at the dusk-side magnetopause flank (Eriksson et al., 2009). For reconnection events, the ideal-MHD reconstruction method has the advantage that it can be performed in the X-line reference frame (Teh and Sonnerup, 2008; Teh et al., 2009), whereas the earlier GSbased reconstructions were performed in the deHoffmannTeller (HT) frame, in which the flows are field-aligned and the $\mathrm{X}$-line is in rapid motion.

In ideal-MHD reconstruction, the magnetic field is frozen in the plasma, i.e., $\boldsymbol{E}+\boldsymbol{v} \times \boldsymbol{B}=0$, and it is only numerical effects that allow reconnection to occur. However, in real reconnection events the frozen-in condition is for physical reasons invalid in the ion and electron diffusion regions. The mechanisms that break the frozen-in condition within these regions are thought to be a combination of, or the separate effects of, resistivity, Hall physics, and electron pressure tensor and inertia. In the present paper, only resistivity will be included. For detailed studies of the ion diffusion region, Sonnerup and Teh (2009) have developed a 2-D reconstruction method for ideal and resistive Hall MHD. They derived an exact axi-symmetric solution and benchmarked the numerical code for the case of ideal Hall MHD, with Ohm's law in the form $\boldsymbol{E}+\boldsymbol{v} \times \boldsymbol{B}=(1 / n e)\left(\boldsymbol{j} \times \boldsymbol{B}+\nabla p_{e}\right)$. For resistive Hall MHD, the reconstruction algorithm also exists but, for lack of a suitable axis-symmetric solution, has yet to be benchmarked. From the reconstruction algorithm for resistive Hall MHD, we can obtain the case of resistive MHD by simply omitting the Hall current and electron pressure terms so that Ohm's law reduces to $\boldsymbol{E}+\boldsymbol{v} \times \boldsymbol{B}=\boldsymbol{\eta} \cdot \boldsymbol{j}$.

In the present paper, we present an exact axi-symmetric solution for resistive MHD and use it to validate the numerical code. We then apply resistive MHD reconstruction to a synthetic reconnection event from a 3-D resistive MHD simulation, where the resistivity is spatially localized and timeindependent. The configuration to be reconstructed is only weakly time dependent and the deviations from 2-D are also weak (Denton et al., 2010).

The layout of the present paper is as follows. In Sect. 2 the basic equations for steady, 2-D, resistive MHD are given. The reconstruction method itself is briefly described in Sect. 3. In Sect. 4, an exact axi-symmetric solution for resistive MHD is presented and used to benchmark the code. In Sect. 5, the resistive code is applied to the simulated reconnection event. Section 6 contains a summary and discussion of the reconstruction results.

\section{Basic equations}

The time-independent equations used for the resistive MHD reconstruction are:

$\nabla \cdot \rho \boldsymbol{v}_{\perp}=0$,

$\rho \boldsymbol{v}_{\perp} \cdot \nabla \boldsymbol{v}=-\nabla p+\boldsymbol{j} \times \boldsymbol{B}$, $\frac{p}{\gamma-1} \boldsymbol{v}_{\perp} \cdot \nabla \ln \left(p / \rho^{\gamma}\right)=\boldsymbol{j} \cdot \boldsymbol{\eta} \cdot \boldsymbol{j}$

$\nabla \cdot \boldsymbol{B}_{\perp}=0$,

$\nabla \times \boldsymbol{E}=0$

$\nabla \times \boldsymbol{B}=\mu_{0} \boldsymbol{j}$,

$\boldsymbol{E}+\boldsymbol{v} \times \boldsymbol{B}=\boldsymbol{\eta} \cdot \boldsymbol{j}$,

where the subscript $\perp$ denotes vector components confined to the $x-y$-plane (the reconstruction plane), i.e., the plane perpendicular to the invariant axis $\hat{z}$, along which axis $\partial / \partial z=0$, so that $\nabla=\hat{x} \partial / \partial x+\hat{y} \partial / \partial y$. The first three equations are the conservation laws for mass, momentum, and entropy, respectively. Equations (1) and (4) can be identically satisfied by writing $\rho \boldsymbol{v}_{\perp}=\nabla \psi \times \hat{z}$ and $\boldsymbol{B}_{\perp}=\nabla A \times \hat{z}$, where $\psi(x, y)$ and $A(x, y)$ are the compressible stream function and vector potential, respectively. Note that, in general, axial fields and flows are included, i.e., $\boldsymbol{B}=\boldsymbol{B}_{\perp}+B_{Z}(x, y) \hat{z}$ and $\boldsymbol{v}=\boldsymbol{v}_{\perp}+v_{\mathrm{z}}(x, y) \hat{z}$. The electric field is given by $\boldsymbol{E}=$ $-\nabla \phi(x, y)+E_{\mathrm{z} 0} \hat{z}$, which satisfies Eq. (5). Here $E_{\mathrm{z} 0}$ is the constant axial electric field and $\phi$ is the electric potential describing the field components in the reconstruction plane. We assume the resistivity tensor to be diagonal in the reconstruction coordinate system but allow for two different values of resistivity, $\eta_{\perp}$ for currents in the reconstruction plane, and $\eta_{\mathrm{z}}$ for those in the axial direction, so that $\boldsymbol{\eta} \cdot \boldsymbol{j}=\eta_{\perp} \boldsymbol{j}_{\perp}+\eta_{\mathrm{z}} j_{\mathrm{z}} \hat{z}$. In our paper, this choice is made for mathematical and computational convenience. In reality, the resistivity tensor is likely to be organized to a considerable extent by the complicated magnetic field near the reconnection site and, in this field, the invariant direction plays an important role. Note that the two resistivities $\eta_{\perp}$ and $\eta_{\mathrm{z}}$ could be assumed as functions of the current densities $\left|\boldsymbol{j}_{\perp}\right|$ and $\left|j_{z}\right|$, respectively. At the present stage, we assume that they are spatially uniform in our studies. The current density can be written as $\boldsymbol{j}=\left(1 / \mu_{0}\right)\left[\nabla B_{\mathrm{Z}} \times \hat{z}-\hat{z} \nabla^{2} A\right]$ so that $\boldsymbol{j} \times \boldsymbol{B}=-\left(1 / \mu_{0}\right)\left[\nabla B_{\mathrm{z}}^{2} / 2+\left(\nabla^{2} A\right) \nabla A+\nabla A \times \nabla B_{\mathrm{z}}\right]$.

Using the above expressions, Eqs. (2), (3), and (7) give the following set:

$$
\begin{aligned}
& \rho\left(v_{\mathrm{x}} \partial / \partial x+v_{\mathrm{y}} \partial / \partial y\right)[(1 / \rho) \partial \psi / \partial y] \\
& \quad=-(\partial / \partial x)\left[p+B_{\mathrm{z}}^{2} / 2 \mu_{0}\right]+\left(B_{\mathrm{y}} / \mu_{0}\right) \nabla^{2} A
\end{aligned}
$$

$$
\begin{aligned}
& \rho\left(v_{\mathrm{x}} \partial / \partial x+v_{\mathrm{y}} \partial / \partial y\right) v_{\mathrm{y}} \\
& \quad=-(\partial / \partial y)\left[p+B_{\mathrm{z}}^{2} / 2 \mu_{0}\right]-\left(B_{\mathrm{x}} / \mu_{0}\right) \nabla^{2} A,
\end{aligned}
$$

$\rho\left(v_{\mathrm{x}} \partial / \partial x+v_{\mathrm{y}} \partial / \partial y\right) v_{\mathrm{z}}=\left(1 / \mu_{0}\right)\left(B_{\mathrm{x}} \partial / \partial x+B_{\mathrm{y}} \partial / \partial y\right) B_{\mathrm{z}},(10)$

$E_{\mathrm{x}}{ }^{\prime \prime} \equiv E_{\mathrm{x}}+v_{\mathrm{y}} B_{\mathrm{z}}-v_{\mathrm{z}} B_{\mathrm{y}}=\frac{\eta_{\perp}}{\mu_{0}} \partial B_{\mathrm{z}} / \partial y$,

$E_{\mathrm{y}}{ }^{\prime \prime} \equiv E_{\mathrm{y}}+v_{\mathrm{z}} B_{\mathrm{x}}-v_{\mathrm{x}} B_{\mathrm{z}}=-\frac{\eta_{\perp}}{\mu_{0}} \partial B_{\mathrm{z}} / \partial x$, 
$E_{\mathrm{z}}^{\prime \prime} \equiv E_{\mathrm{z} 0}+v_{\mathrm{x}} B_{\mathrm{y}}-v_{\mathrm{y}} B_{\mathrm{x}}=-\frac{\eta_{\mathrm{z}}}{\mu_{0}} \nabla^{2} A$

$$
\begin{gathered}
\frac{p v_{\mathrm{x}}}{\gamma-1} \frac{\partial}{\partial x}\left(\ln \frac{p}{\rho^{\gamma}}\right)-\frac{\gamma p v_{\mathrm{y}}}{(\gamma-1) \rho} \partial \rho / \partial y+\frac{v_{\mathrm{y}}}{\gamma-1} \partial p / \partial y \\
=\frac{\eta_{\perp}}{\mu_{0}^{2}}\left[\left(\frac{\partial B_{\mathrm{z}}}{\partial x}\right)^{2}+\left(\frac{\partial B_{\mathrm{z}}}{\partial y}\right)^{2}\right]+\frac{\eta_{\mathrm{z}}}{\mu_{0}^{2}}\left(\nabla^{2} A\right)^{2}
\end{gathered}
$$

where $\nabla^{2} A=\partial^{2} A / \partial x^{2}+\partial^{2} A / \partial y^{2}$. Equations (8) to (14) can be obtained from the equations in the paper of Sonnerup and Teh (2009) by neglecting the electron pressure and Hall current terms in the momentum equation and Ohm's law. In the next section, Eqs. (8) to (14) will be used as the basis for the reconstruction code.

\section{Reconstruction method}

To perform the reconstruction, direct measurements of the electric field are required. If only two components of the electric field, usually those in the spacecraft spin-plane, are measured, a specified resistivity model is then needed for the reconstruction, as discussed in detail in Appendix B of Sonnerup and Teh (2009), in which the Hall current and electron pressure terms are to be deleted for our present purposes. In what follows, we apply the resistive MHD reconstruction method under the assumption that all three electric field components have been measured.

Before doing the reconstruction, the invariant axis $\hat{z}$ and the moving frame velocity must be determined, which is a nontrivial task. A single-spacecraft method, based on the constancy of the axial electric field component, $E_{\mathrm{z} 0}$, in 2-D, steady field configurations, exists (Sonnerup and Hasegawa, 2005) but does not work when the electric field is nearly unidirectional. For further discussion, see the papers on ideal MHD reconstruction (Sonnerup and Teh, 2008; Teh and Sonnerup, 2008, 2009). Use of multi-spacecraft information is often needed and will be employed here.

The vector potential $A(x, y=0)$ and compressible stream function $\psi(x, y=0)$ can be calculated by integration along the $\mathrm{x}$-axis, which is chosen as the projection of the spacecraft path onto the reconstruction plane:

$$
\begin{aligned}
& A(x, 0)=-\int_{x^{\prime}=0}^{x^{\prime}=x} B_{\mathrm{y}}\left(x^{\prime}, 0\right) d x^{\prime}, \\
& \psi(x, 0)=-\int_{x^{\prime}=0}^{x^{\prime}=x} \rho\left(x^{\prime}, 0\right) v_{\mathrm{y}}\left(x^{\prime}, 0\right) d x^{\prime} .
\end{aligned}
$$

In our 2-D geometry, all physical quantities and their $\mathrm{x}$ derivatives are known at points on the $\mathrm{x}$-axis $(y=0)$. Away from $y=0$, the values $A(x, y)$ and $\psi(x, y)$ are calculated by use of Taylor expansions to second order, i.e.,

$$
\begin{aligned}
A(x, y \pm \Delta y)= & A(x, y) \pm \Delta y \partial A(x, y) / \partial y \\
& +1 / 2(\Delta y)^{2} \partial^{2} A(x, y) / \partial y^{2}, \\
\psi(x, y \pm \Delta y)= & \psi(x, y) \pm \Delta y \partial \psi(x, y) / \partial y \\
& +1 / 2(\Delta y)^{2} \partial^{2} \psi(x, y) / \partial y^{2},
\end{aligned}
$$

where $\partial A / \partial y=B_{\mathrm{x}}$ and $\partial \psi / \partial y=\rho v_{\mathrm{x}}$.

To determine the resistivity, we can use Eq. (12) to estimate the value of $\eta_{\perp}$ and then assume that $\eta_{\mathrm{z}}=k \eta_{\perp}$, where $k$ is a chosen constant. (The special case where $\eta_{\perp}=0$ and $\eta_{\mathrm{z}} \neq 0$ is also doable but requires a somewhat different procedure.) After the resistivity has been determined, the values of $y$-derivatives (e.g., $\partial B_{\mathrm{z}} / \partial y, \partial v_{\mathrm{z}} / \partial y$, etc.) can be estimated in sequence as follows:

1. The values of $\partial B_{\mathrm{z}} / \partial y$ and $\partial^{2} A / \partial y^{2}$ are calculated from Eqs. (11) and (13), respectively.

2. The value of $\partial v_{z} / \partial y$ is obtained by substituting the value of $\partial B_{z} / \partial y$ into Eq. (10).

3. The derivatives $\partial \rho / \partial y$ and $\partial p / \partial y$ are calculated by solving Eqs. (9) and (14) together, using the known values of $\partial B_{\mathrm{z}} / \partial y$ and $\partial^{2} A / \partial y^{2}$, along with mass conservation, which requires $\partial v_{\mathrm{y}} / \partial y=-\partial v_{\mathrm{x}} / \partial x-$ $(1 / \rho)\left(v_{\mathbf{x}} \partial \rho / \partial x+v_{\mathrm{y}} \partial \rho / \partial y\right)$.

4. The values of $\partial \rho / \partial y$ and $\partial^{2} A / \partial y^{2}$ can be substituted into Eq. (8) to give $\partial^{2} \psi / \partial y^{2}$.

The calculation of the values of $\partial v_{\mathrm{z}} / \partial y$ and $\partial^{2} \psi / \partial y^{2}$ requires that the velocity component $v_{\mathrm{y}} \neq 0$. At locations where $v_{\mathrm{y}}=0$, we can use nearby grid points and a spline function to obtain the values of the derivatives by interpolation.

With the known values of these $\mathrm{y}$-derivatives, the quantities $\left(\rho, p, v_{\mathrm{z}}, B_{\mathrm{z}}\right)$ can be advanced at each step, e.g., $\rho(x, y \pm$ $\Delta y)=\rho(x, y) \pm \Delta y \partial \rho / \partial y$. By use of Eq. (5), we find that $\partial E_{\mathrm{x}} / \partial y=\partial E_{\mathrm{y}} / \partial x$ so that the new electric field component along the $\mathrm{x}$-axis becomes $E_{\mathrm{X}}(x, y \pm \Delta y)=E_{\mathrm{x}}(x, y) \pm$ $\Delta y \partial E_{\mathrm{y}} / \partial x$, where $\partial E_{\mathrm{y}} / \partial x$ is known at each step. The axial electric field component $E_{\mathrm{z} 0}$ remains unchanged and, finally, the new set of values of $E_{\mathrm{y}}(x, y \pm \Delta y)$ are obtained by substituting the values of $v_{\mathrm{x}}, v_{\mathrm{Z}}, B_{\mathrm{X}}$, and $B_{\mathrm{Z}}$ at $y=y \pm \Delta y$ into Eq. (12).

\section{Benchmark case}

Our axi-symmetric $(\partial / \partial \varphi=0)$ exact solution for steady, 2-D $(\partial / \partial z=0)$, resistive MHD is similar to the case presented in Appendix A of Sonnerup and Teh (2009), except that the Hall term and electron pressure are eliminated and the resistivity is included. The exact solution depends only on the 
cylindrical radius $r$. It is developed in a coordinate system that rotates at a constant angular rate $\omega$ around the invariant axis $\hat{z}$, and slides at constant velocity $-\tilde{V}_{z 0} \hat{z}$ along that axis. In the rotating frame, the momentum equation is expressed as

$\rho \boldsymbol{v}^{\prime} \cdot \nabla \boldsymbol{v}^{\prime}=-\nabla p+\boldsymbol{j} \times \boldsymbol{B}+2 \rho \boldsymbol{v}^{\prime} \times \omega+\rho \boldsymbol{r} \omega^{2}$,

where the prime denotes velocities evaluated in the frame that is rotating and sliding along the $\hat{z}$ direction. Four exact integrals can be obtained from the conservation of magnetic flux, mass, angular momentum, and $z$-momentum:

$\tilde{B}_{r}=\tilde{B}_{r 0} / \tilde{r}$

$\tilde{v}_{r}^{\prime}=\tilde{v}_{r}=\tilde{v}_{r 0} / \tilde{r} \tilde{\rho}$,

$\tilde{v}_{\varphi}^{\prime}=\kappa M_{A 0}^{-2} \tilde{B}_{\varphi}-\Omega \tilde{r}+C_{\varphi} / \tilde{r}$

$\tilde{v}_{\mathrm{z}}^{\prime}=\kappa M_{A 0}^{-2} \tilde{B}_{\mathrm{Z}}+C_{\mathrm{z}}$

where $\kappa=\tilde{B}_{r 0} / \tilde{v}_{r 0}, \Omega=\omega r_{0} / v_{0}, M_{A 0}=v_{0}\left(\mu_{0} \rho_{0}\right)^{1 / 2} / B_{0}$, $C_{\varphi}=\tilde{v}_{\varphi 0}^{\prime}-\kappa M_{A 0}^{-2} \tilde{B}_{\varphi 0}+\Omega$, and $C_{\mathrm{z}}=\tilde{v}_{\mathrm{z} 0}^{\prime}-\kappa M_{A 0}^{-2} \tilde{B}_{\mathrm{z} 0}$. Note that non-dimensional variables are denoted by a tilde; their reference values are $\left(r_{0}, \rho_{0}, p_{0}, v_{0}, B_{0}\right)$, where $v_{0}^{2}=v_{r 0}^{2}+$ $v_{\varphi 0}^{\prime 2}+v_{\mathrm{z} 0}^{\prime 2}$ and $B_{0}^{2}=B_{r 0}^{2}+B_{\varphi 0}^{2}+B_{\mathrm{z} 0}^{2}$. The radial components of Eq. (19) and the entropy law are:

$$
\begin{aligned}
& \frac{\tilde{v}_{r 0}^{2}}{\tilde{r}^{2} \tilde{\rho}^{2}} \frac{d \tilde{\rho}}{d \tilde{r}}-\frac{1}{\gamma} M_{s 0}^{-2} \frac{d \tilde{p}}{d \tilde{r}}=M_{A 0}^{-2} \tilde{B}_{\mathrm{Z}} \frac{d \tilde{B}_{\mathrm{Z}}}{d \tilde{r}}+M_{A 0}^{-2} \frac{\tilde{B}_{\phi}}{\tilde{r}} \frac{d \tilde{r} \tilde{B}_{\phi}}{d \tilde{r}} \\
& -\frac{\tilde{\rho}}{\tilde{r}}\left(\kappa M_{A 0}^{-2} \tilde{B}_{\phi}+\frac{C_{\phi}}{\tilde{r}}\right)^{2}-\frac{\tilde{v}_{r 0}^{2}}{\tilde{r}^{3} \tilde{\rho}}, \\
& -\frac{1}{(\gamma-1)} M_{s 0}^{-2} \frac{\tilde{p}}{\tilde{\rho}} \frac{d \tilde{\rho}}{d \tilde{r}}+\frac{1}{\gamma(\gamma-1)} M_{s 0}^{-2} \frac{d \tilde{p}}{d \tilde{r}} \\
& \quad=\tilde{v}_{r 0}^{-1} M_{A 0}^{-2} \tilde{\rho} \tilde{r}\left(\tilde{\eta}_{\perp} \tilde{j}_{\phi}^{2}+\tilde{\eta}_{z} \tilde{j}_{\mathrm{Z}}^{2}\right),
\end{aligned}
$$

where $M_{s 0}=v_{0}\left(\rho_{0} / \gamma p_{0}\right)^{1 / 2}$. Also, the resistivities and current densities have been made non-dimensional using the normalization constants, $\mu_{0} v_{0} r_{0}$ and $B_{0} / \mu_{0} r_{0}$, respectively.

In the rotating (primed) frame, the electric field $\boldsymbol{E}^{\prime}$ is assumed to be purely radial. Also, the radial current density $j_{\mathrm{r}}$ must be equal to zero in our special geometry, in which $\partial / \partial \phi=0$ and $\partial / \partial z=0$. The angular and z-components of the Ohm's law equation can be written as:

$\tilde{v}_{\mathrm{z}}^{\prime} \tilde{B}_{\mathrm{r}}-\tilde{v}_{\mathrm{r}} \tilde{B}_{\mathrm{Z}}=\tilde{\eta}_{\perp} \tilde{j}_{\varphi}=-\tilde{\eta}_{\perp} \frac{d \tilde{B}_{\mathrm{Z}}}{d \tilde{r}}$,

and

$\tilde{v}_{\mathrm{r}} \tilde{B}_{\varphi}-\tilde{v}_{\varphi}^{\prime} \tilde{B}_{\mathrm{r}}=\tilde{\eta}_{\mathrm{z}} \tilde{j}_{\mathrm{Z}}=\tilde{\eta}_{\mathrm{z}} \frac{1}{\tilde{r}} \frac{d\left(\tilde{r} \tilde{B}_{\varphi}\right)}{d \tilde{r}}$.

By substituting Eqs. (20)-(23) into Eqs. (26) and (27), we can get

$\tilde{j}_{\phi}=-\frac{d \tilde{B}_{\mathrm{Z}}}{d \tilde{r}}=\frac{1}{\tilde{\eta}_{\perp}}\left(\frac{\tilde{B}_{r 0}}{\tilde{r}}\left(\kappa M_{A 0}^{-2} \tilde{B}_{\mathrm{Z}}+C_{\mathrm{z}}\right)-\tilde{v}_{\mathrm{r} 0} \frac{1}{\tilde{r} \tilde{\rho}} \tilde{B}_{\mathrm{Z}}\right)$,

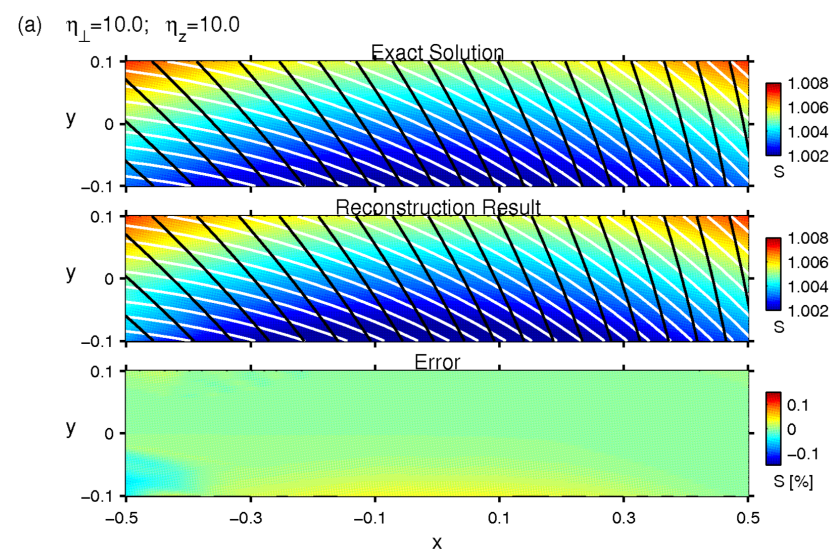

(b) $\eta_{\perp}=1.0 ; \eta_{z}=0.5$

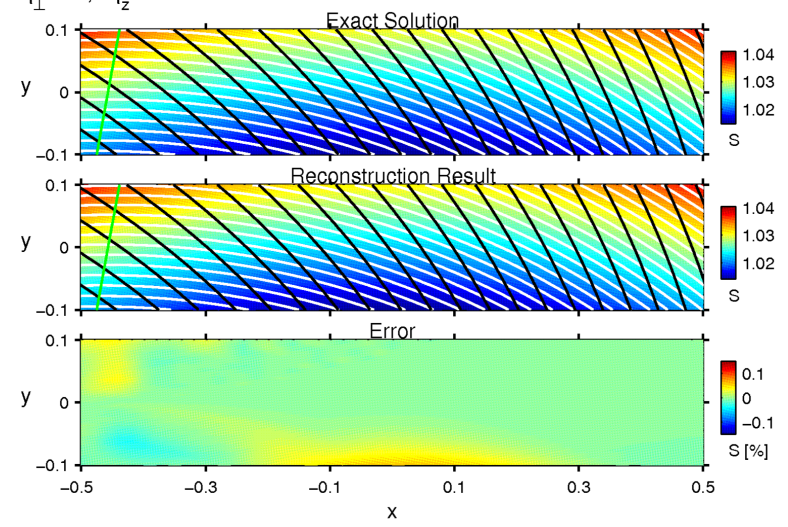

Fig. 1. Comparison of exact benchmark solution with reconstruction, for (a) $\tilde{\eta}_{\perp}=\tilde{\eta}_{\mathrm{z}}=10.0$, and (b) $\tilde{\eta}_{\perp}=1.0, \tilde{\eta}_{\mathrm{z}}=0.5$. The top panel shows the overlay of the exact magnetic field lines (black) and streamlines (white) with entropy $S=p / \rho^{\gamma}$ in color, while the middle panel shows the corresponding reconstruction results. The error distribution for entropy $S$, expressed in percent of the average of $|S|$ over the reconstruction window, is shown in the bottom panel. The green line on the left in the first two panels of Fig. 1b indicates the locations where $v_{\mathrm{y}}=0$.

$\tilde{j}_{\mathrm{Z}}=\frac{1}{\tilde{r}} \frac{d \tilde{r} \tilde{B}_{\phi}}{d \tilde{r}}=\frac{1}{\tilde{\eta}_{\mathrm{z}}}\left(\tilde{v}_{\mathrm{r} 0} \frac{\tilde{B}_{\phi}}{\tilde{r} \tilde{\rho}}-\tilde{B}_{\mathrm{r} 0} \frac{1}{\tilde{r}}\left(\kappa M_{A 0}^{-2} \tilde{B}_{\phi}-\Omega \tilde{r}+\frac{C_{\phi}}{\tilde{r}}\right)\right)$.

With a specified resistivity, we can now solve Eqs. (24) and (25) for $d \tilde{\rho} / d \tilde{r}$ and $d \tilde{p} / d \tilde{r}$ by use of Eqs. (28) and (29). The actual integration of the system is performed by a simple outward marching procedure, starting at $\tilde{r}=1$ where the flow is taken to be field-aligned, i.e., $\left(\tilde{v}_{\mathrm{r} 0}, \tilde{v}_{\varphi 0}^{\prime}, \tilde{v}_{\mathrm{z} 0}^{\prime}\right)=$ $\left(\tilde{B}_{r 0}, \tilde{B}_{\varphi 0}, \tilde{B}_{\mathrm{Z} 0}\right)$. The quantities $\left(\tilde{\rho}, \tilde{p}, \tilde{B}_{\varphi}, \tilde{B}_{\mathrm{z}}\right.$, etc.) are then advanced to first order so that, for example, $\tilde{\rho}(\tilde{r}+\Delta \tilde{r})=$ $\tilde{\rho}(\tilde{r})+\Delta \tilde{r} d \tilde{\rho} / d \tilde{r}$. The final step is to revert to the fixed frame in which the velocity components along $\hat{\varphi}$ and $\hat{z}$ are transformed as

$\tilde{v}_{\varphi}=\tilde{v}_{\varphi}^{\prime}+\Omega \tilde{r} ; \quad \tilde{v}_{\mathrm{Z}}=\tilde{v}_{\mathrm{z}}^{\prime}+\tilde{V}_{\mathrm{z} 0}$. 
Also, the electric field components (normalized to $v_{0} B_{0}$ ) change to:

$$
\begin{aligned}
\tilde{E}_{\mathrm{r}} & =\tilde{E}_{\mathrm{r}}^{\prime}-\Omega \tilde{r} \tilde{B}_{\mathrm{Z}}+\tilde{V}_{\mathrm{z} 0} \tilde{B}_{\varphi} ; \\
\tilde{E}_{\varphi} & =-\tilde{V}_{\mathrm{z} 0} \tilde{B}_{\mathrm{r}} ; \quad \tilde{E}_{\mathrm{z}}=\Omega \tilde{r} \tilde{B}_{\mathrm{r}}=\Omega \tilde{B}_{\mathrm{r} 0}=\text { const. } \equiv \tilde{E}_{\mathrm{z} 0} .
\end{aligned}
$$

The overlay maps of magnetic field lines (black) and streamlines (white), with pseudo-entropy $S=p / \rho^{\gamma}$ in color, are shown in the top panel of each of the two panel blocks in Fig. 1, for the exact solution with (a) $\tilde{\eta}_{\perp}=\tilde{\eta}_{\mathrm{z}}=10.0$ and (b) $\tilde{\eta}_{\perp}=1.0, \tilde{\eta}_{\mathrm{Z}}=0.5$. The non-dimensional parameters used are: $M_{A 0}=0.45, M_{s 0}=0.40, \Omega=0.875$, $\left(\tilde{B}_{r 0}^{2}, \tilde{B}_{\varphi 0}^{2}, \tilde{B}_{\mathrm{z} 0}^{2}\right)=\left(\tilde{v}_{r 0}^{\prime 2}, \tilde{v}_{\varphi 0}^{\prime 2}, \tilde{v}_{\mathrm{z} 0}^{\prime 2}\right)=(0.50,0.15,0.35), \quad \tilde{V}_{\mathrm{z} 0}=$ 1.0 , and $\gamma=5 / 3$. Note that the constant axial electric field $\tilde{E}_{\mathrm{z} 0}$ is 0.62 for both cases. The data taken from the exact solution along the line $y=0$ are used as initial values for the reconstruction, the results of which are shown in the middle panel of each panel block. Error distribution maps are in the third panel of each block. Within the reconstruction domain, the errors are less than $1.0 \%$ for all quantities, including those not shown in the paper. For the case in panel (b) of Fig. 1, the green line shown in the first two panels indicates locations where $v_{\mathrm{y}}=0$. The results indicate that the interpolation is doing well at locations fairly close to the line $y=0$ (the spacecraft path), but that the errors start to grow when the integration is extended to locations further away. The overall results show that the reconstruction method is able to recover the field and plasma configurations accurately for a relatively narrow domain, elongated along the line $y=0$. We have also performed experiments for various numerical parameters, namely, doubling the grid resolution and extending the domain to $50 \%$ larger $|y|$ value. The results for the errors are summarized in Table 1.

From the benchmarking exercises described above, we conclude that the numerical code functions properly and gives accurate results in an elongated region surrounding the data line at $y=0$, provided the base assumptions of the reconstruction, namely that the object reconstructed is 2-D and time independent, are precisely satisfied.

\section{Case study: simulated magnetotail reconnection event}

We now apply the resistive MHD reconstruction code to a synthetic reconnection event in the geomagnetic tail, obtained from a 3-D, resistive MHD simulation (e.g., Birn et al., 2009). In the simulation model, the resistivity, assumed isotropic, is given by

$\eta=\eta_{0} / \cosh s$,

where $s=\left[\left(\left(x^{\prime}-x_{0}\right) / L_{\mathrm{x}}\right)^{2}+\left(y^{\prime} / L_{\mathrm{y}}\right)^{2}+\left(z^{\prime} / L_{\mathrm{z}}\right)^{2}\right]^{1 / 2}$ and $\eta_{0}, z_{0}, L_{\mathrm{x}}, L_{\mathrm{y}}$, and $L_{\mathrm{z}}$ are non-dimensional constants. The reference values used for the simulation are $L_{0}=1000 \mathrm{~km}$,

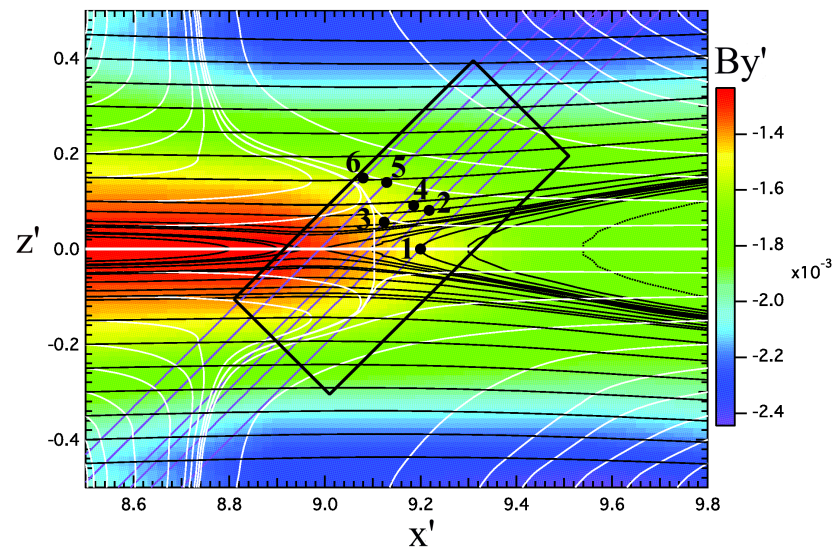

Fig. 2. Plot of magnetic field lines (black) and streamlines (white) in the $x^{\prime}-z^{\prime}$ plane of the simulation, with the extremely weak, normalized (in units of $20 \mathrm{nT}$ ) field $B_{y^{\prime}}$ in that plane shown in color. Also shown are six simulated spacecraft paths through the vicinity of the reconnection site. The spacecraft paths are in reality inclined by $19.5^{\circ}$ to the $x^{\prime}-z^{\prime}$ plane. The simulated spacecraft $1-4$ form a tetrahedron configuration while the spacecraft 5 and 6 are used as auxiliaries for verification of the reconstruction results. The region to be reconstructed is within the black box. In the simulation, the $y^{\prime}$-axis is the axis of approximate invariance.

$W_{0}=1000 \mathrm{~km} \mathrm{~s}^{-1}$, and $B_{0}=20 \mathrm{nT}$, for the length, the velocity, and the magnetic field, respectively, and the resistivity is normalized by $\mu_{0} W_{0} L_{0}$. In the expression for $s$, the $x^{\prime}$ and $y^{\prime}$ axes are directed tailward and duskward, respectively, and $z^{\prime}=x^{\prime} \times y^{\prime}$. We see that the resistivity is spatially localized and time-independent. Figure 2 shows the magnetic field line and streamline configurations in the $x^{\prime}-z^{\prime}$ plane, with the axial field $B_{y^{\prime}}$ in color, from the simulation, along with six simulated spacecraft paths through the vicinity of the reconnection site. The axial field is seen to be extremely weak. Field lines and streamlines were obtained by direct integration, using the simulated field and flow components in the plane. In the simulation, the values $\eta_{0}=0.05$, $x_{0}=8, L_{\mathrm{x}}=4, L_{\mathrm{y}}=8$, and $L_{\mathrm{z}}=1$ were used. The simulated spacecraft $1-4$ form a tetrahedron configuration, while the spacecraft 5 and 6 are used as auxiliaries for verification of the reconstruction results. Figure 3 shows the magnetic field, electric field, and plasma data for all six simulated spacecraft; the reconstruction interval is sandwiched between the two gray vertical lines. Note that the simulation data were all taken at a single instant and therefore comprise snapshot information about the structure. The coordinate system used in Fig. 3 was intentionally rotated by one of us (J. B.) in a way that was unknown to the other authors who analyzed the results, and the rotated data was used in the reconstruction. The purpose of this blind test was to see if the orientation of the invariant direction, as well as any motion of the X-line, could be independently deduced from the spacecraft data. In the rotated coordinates, the positions 
Table 1. Reconstruction errors ${ }^{\mathrm{a}}$.

\begin{tabular}{lccc}
\hline & \multicolumn{3}{c}{ Resistive MHD } \\
& $\left(M_{A 0}=0.45, M_{S 0}=0.40, \Omega=0.875, \tilde{V}_{\mathrm{z} 0}=1.0, \gamma=5 / 3\right.$, \\
& \multicolumn{3}{c}{$\left.=\tilde{\eta}_{\mathrm{z}}=10.0\right)$} \\
\cline { 2 - 4 } & Grid $\left(n_{\mathrm{X}} / n_{\mathrm{y}}\right)$ & Grid $\left(n_{\mathrm{X}} / n_{\mathrm{y}}\right)$ & Grid $\left(n_{\mathrm{X}} / n_{\mathrm{y}}\right)$ \\
& $51 / 101$, & $101 / 201$, & $101 / 301$, \\
& Window $\left(2 l_{\mathrm{y}} / l_{\mathrm{X}}\right)$ & Window $\left(2 l_{\mathrm{y}} / l_{\mathrm{X}}\right)$ & Window $\left(2 l_{\mathrm{y}} / l_{\mathrm{X}}\right)$ \\
& $0.20^{\mathrm{b}}$ & $0.20^{\mathrm{c}}$ & $0.30^{\mathrm{d}}$ \\
\hline Error $A(\%)$ & 0.014 & 0.005 & 0.010 \\
Error $\psi(\%)$ & 0.030 & 0.011 & 0.019 \\
Error $B_{\mathrm{Z}}(\%)$ & 0.001 & 0.000 & 0.000 \\
Error $v_{\mathrm{Z}}(\%)$ & 0.002 & 0.001 & 0.002 \\
Error $S(\%)$ & 0.013 & 0.012 & 0.057 \\
Error $E_{\mathrm{X}}(\%)$ & 0.156 & 0.059 & 0.105 \\
Error $E_{\mathrm{y}}(\%)$ & 0.701 & 0.257 & 0.527 \\
\hline
\end{tabular}

a Average, in domain $-l_{\mathrm{x}} / 2 \leq x \leq l_{\mathrm{x}} / 2$, of error magnitudes at $y= \pm y_{\max } \equiv \pm l_{\mathrm{y}}$ expressed in percent of average magnitude of corresponding quantity (e.g., $\left.B_{\mathrm{Z}}\right)$ over the window. For the test case, $\left(\tilde{B}_{\mathrm{r} 0}^{2}, \tilde{B}_{\varphi 0}^{2}, \tilde{B}_{\mathrm{z} 0}^{2}\right)=\left(\tilde{v}_{r 0}^{2}, \tilde{v}_{\varphi 0}^{2}, \tilde{v}_{\mathrm{z} 0}^{2}\right)=(0.5,0.15,0.35)$.

b Benchmark case shown in Fig. 1a.

${ }^{\mathrm{c}}$ Grid resolution in $x$ and $y$ increased by factor 2 .

$\mathrm{d}$ The larger grid resolution and $l_{\mathrm{y}}$ increased by $50 \%$.
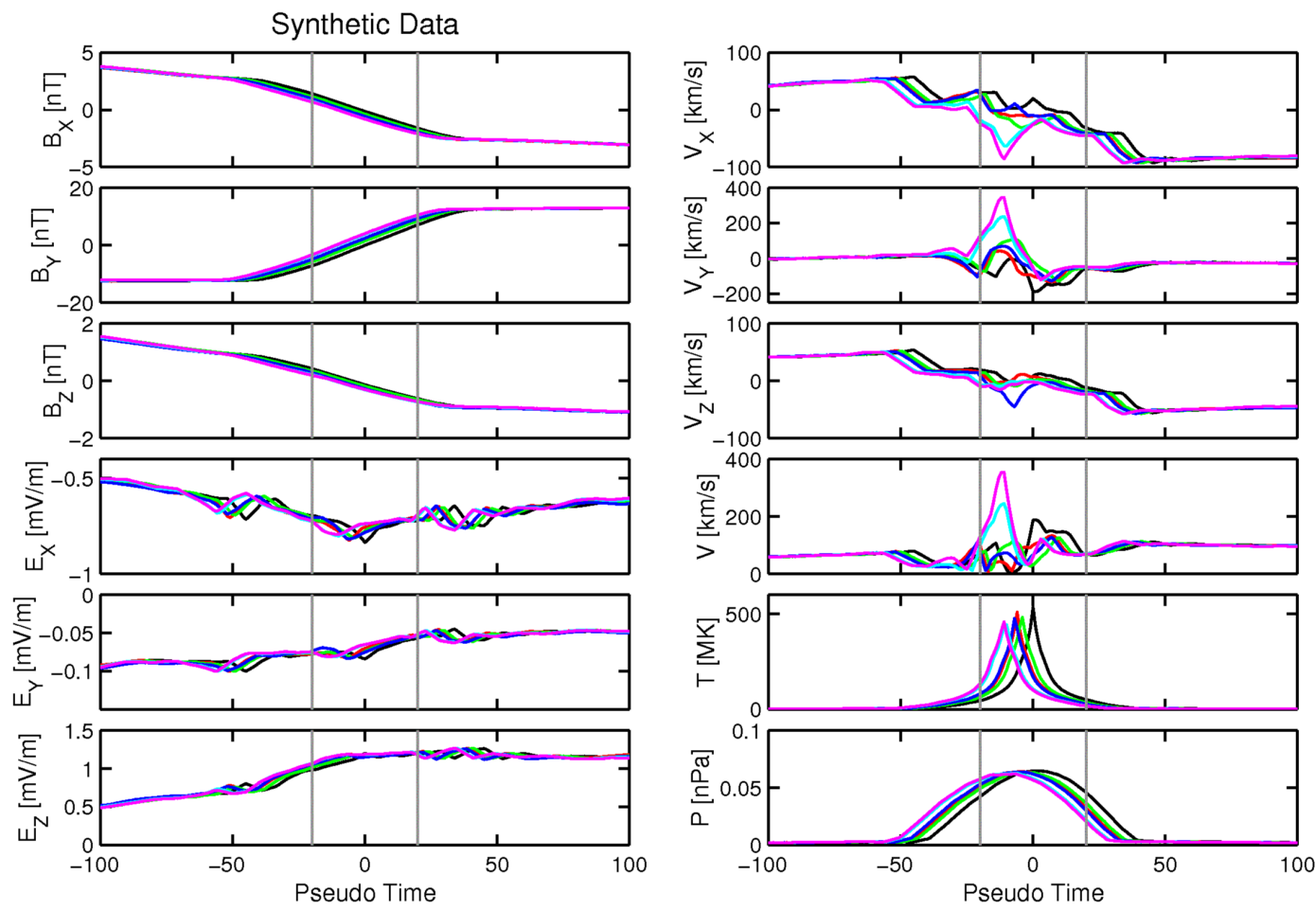

Fig. 3. Plots of the magnetic field, electric field, and plasma data for all six simulated spacecraft. The color used for spacecraft 1 to 6 are black, red, green, blue, cyan, and magenta, respectively. In the rotated coordinates (see text for details), the positions for the spacecraft $1-6$ at $t=0$ are: [0,0,0], [a,0,0], $[1 / 2 a, \sqrt{3 / 4} a, 0],[1 / 2 a, \sqrt{1 / 12} a, \sqrt{2 / 3} a],[3 / 2 a, a, 0]$, and $[3 / 2 a, 3 / 2 a, 0]$, respectively, where $a=100 \mathrm{~km}$. The reconstruction time interval is enclosed by the two vertical lines. 

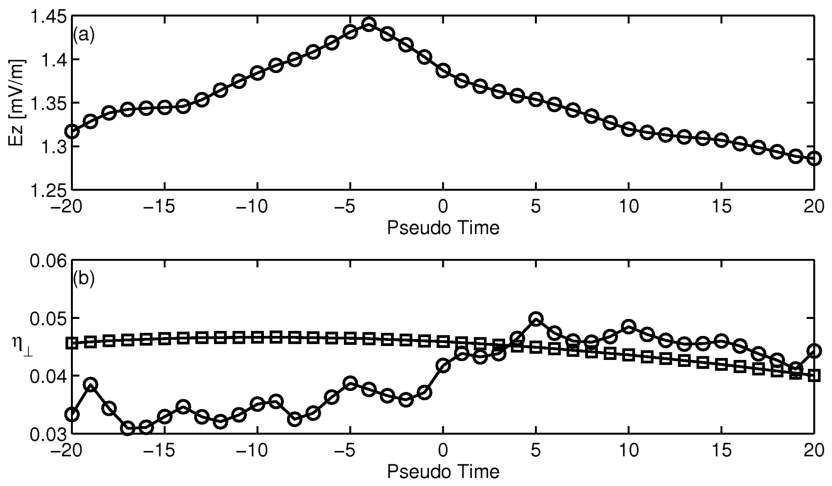

Fig. 4. (a) Plot of the measured axial electric field in the reconstruction interval, evaluated in the moving frame and based on the data from spacecraft 3. (b) Plot of the dimensionless resistivity $\eta_{\perp}$ in the reconstruction interval used in the simulation (squares) and recovered from the reconstruction (circles).

of the spacecraft $1-6$ at some chosen time $t=0$ are: $[0,0,0]$, $[a, 0,0],[1 / 2 a, \sqrt{3 / 4} a, 0],[1 / 2 a, \sqrt{1 / 12} a, \sqrt{2 / 3} a],[3 / 2 a, a, 0]$, and $[3 / 2 a, 3 / 2 a, 0]$, respectively, where $a=100 \mathrm{~km}$. We see that at $t=0$, all spacecraft, except spacecraft 4 , are in the $z=0$ plane of the rotated system. We also know that the spacecraft paths are all parallel but initially we do not know their orientation relative to the rotated coordinate axes. As described in detail in a separate paper (Denton et al., 2010), the multi-spacecraft method developed by Shi et al. (2005, 2006), which is based on the gradient of the magnetic field, was used to estimate the orientation of the invariant axis and the spacecraft motion through the field configuration. In the unknown, rotated coordinates, Denton et al. (private communication, 2010) derived the invariant axis $\hat{z}=[-0.5579,-0.0771,0.8263]$ and the frame velocity $V_{0}=$ $[-16.2,10.0,-3.5] \mathrm{km} \mathrm{s}^{-1}$, which are then used for the reconstruction. Those values agree well with the actual values in the simulation, namely $\hat{z}_{s}=[0.5591,0.0560,-0.8272]$ and $V_{0 s}=[-16.9,10.3,-2.7] \mathrm{km} \mathrm{s}^{-1}$. The frame velocity $V_{0 s}$ has a finite component along $\hat{z}_{s}$, because the spacecraft paths are inclined by $70.5^{\circ}$ to the $\hat{z}_{s}$ axis. By using the recovered axis and velocity instead of the exact values, this angle comes out as $73.9^{\circ}$. Since this is a 3-D simulation model, the invariant axis is defined as the direction along which $\partial \boldsymbol{B} / \partial z$ is minimized, which is the $y^{\prime}$-axis in the simulation coordinates. Note that the results above are based on data from the tetrahedron spacecraft configuration (1-4). The method of Sonnerup and Hasegawa (2005) did not work for this event, because the axial component of the electric field, which is much larger than the components in the reconstruction plane, is in fact not precisely constant, while the in-plane components are small as a consequence of the small axial magnetic field and flow in the simulation and therefore have only small variations.
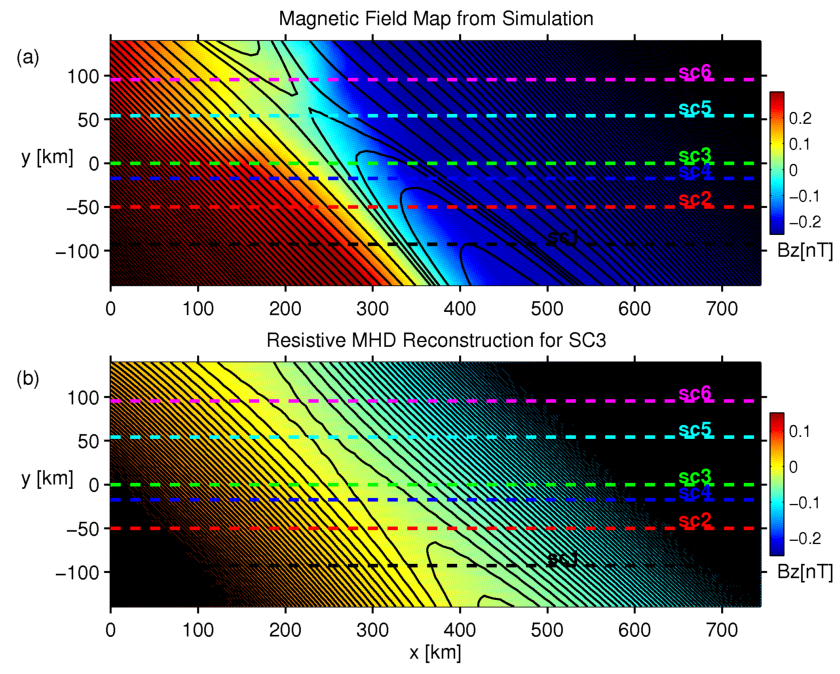

Fig. 5. (a) Plot of magnetic field lines in the reconstruction (x-y) plane, with the axial field $B_{\mathrm{Z}}$ in color. The map is obtained by projection of the simulation results, evaluated in the $x^{\prime}-z^{\prime}$-plane, onto the x-y-plane. The reversal of $B_{\mathrm{Z}}$ across the layer, which is not present in Fig. 2, occurs because the $\mathrm{z}$-axis used in the reconstruction does not coincide exactly with the $\mathrm{y}^{\prime}$-axis in Fig. 2 (they form an angle of $1.3^{\circ}$ ). (b) Resistive MHD reconstruction map of the magnetic field lines (black solid lines) with axial field $B_{\mathrm{Z}}$ in color, based on data measured by spacecraft 3 at points along its path. The horizontal dashed lines are the spacecraft paths, projected onto the reconstruction plane, relative to which they are tilted by $16.1^{\circ}$. Some of the color differences between the maps in panels (a) and (b) are caused by this tilt in combination with the weakly 3-D nature of the simulation. Spacecraft 1-4 form a tetrahedron configuration while spacecraft 5 and 6 are used as auxiliaries for verification of the reconstruction results.

All of the following reconstruction results are based on analysis of the data from the simulated spacecraft 3 . In the reconstruction (as opposed to in the simulation itself), the reference values $b_{0}, n_{0}, v_{0}$, and $l_{0}$ for the magnetic field, the density, the velocity, and the length are, respectively, the maximum value of the magnetic field strength, the maximum value of the density, the Alfvén speed based on the $b_{0}$ and $n_{0}$ values, and the ratio of the maximum value of the vector potential $A$ and the reference field $b_{0}$.

Figure $4 \mathrm{a}$ shows the measured axial $(\hat{z})$ electric field in the reconstruction interval, evaluated in the frame moving with velocity $V_{0}$. One sees that the axial electric field is in fact not precisely constant in the simulation data but varies by about $10 \%$. As a result, the minimum variance direction of the electric field (Sonnerup and Hasegawa, 2005) is not a useful predictor of the invariant axis. The non-constancy of the axial electric field indicates the presence of time-dependent and 3$\mathrm{D}$ effects. In the reconstruction, $E_{\mathrm{z} 0}=1.353 \mathrm{mV} \mathrm{m}^{-1}$ will be used, which is the average value. We use Eq. (12) to estimate the dimensionless values of $\eta_{\perp}$ (normalized by $\mu_{0} v_{0} l_{0}$ ) along the path of spacecraft 3 through the structure. Figure $4 \mathrm{~b}$ 

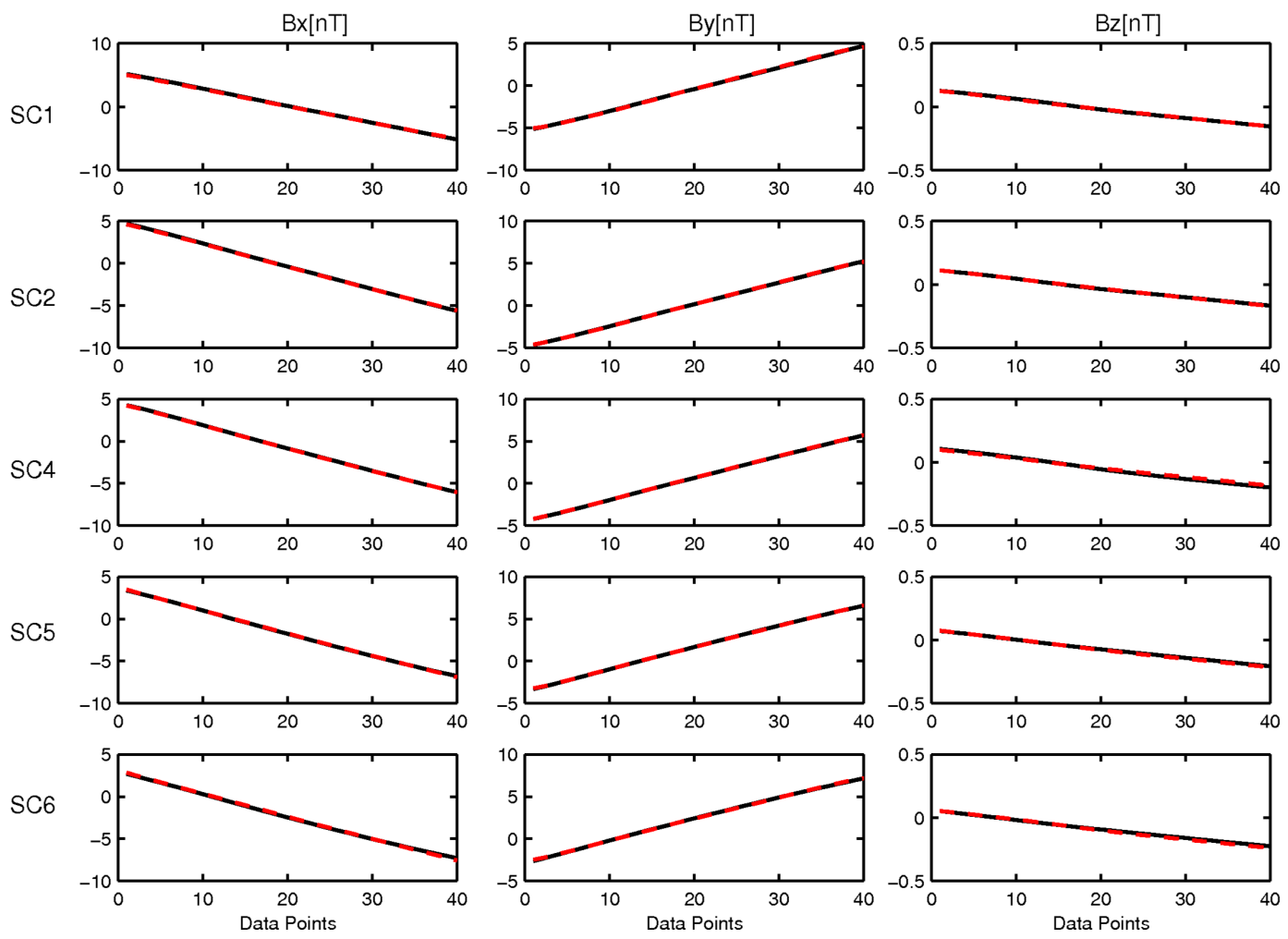

Fig. 6. Snapshot plots of the predicted magnetic fields (red dashed lines) from the reconstruction map based on spacecraft 3 and the corresponding fields (black lines) taken by the other spacecraft directly from the simulation.

shows a plot of dimensionless $\eta_{\perp}$ values in the reconstruction interval, used in the simulation (squares) and recovered from the reconstruction (circles). The agreement between the calculated and the actual values is much better for times $t>0$ than that for $t<0$, but overall, the agreement is not good. This problem arises because the axial magnetic field is very small in our simulation model so that the factor $\partial B_{\mathrm{Z}} / \partial x$ that multiplies $\eta_{\perp}$ in Eq. (12) is also small. By converting time to distance, using the moving frame velocity $V_{0}$, one finds that the resistivity $\eta_{\perp}$ does have a spatial variation with distance along the spacecraft path, even though the variation differs significantly from that in the simulation setup. In the reconstruction, a constant non-dimensional resistivity $\eta_{\perp}=0.05$ will be used, which is the maximum calculated value of $\eta_{\perp}$; we also assume that $\eta_{\mathrm{z}}=\eta_{\perp}$. This choice of the values of $\eta_{\perp}$ and $\eta_{\mathrm{z}}$ will give the best correlation coefficient between the predictions from the reconstruction map and the measurements from the other five simulated spacecraft (which are not otherwise used in the reconstruction). The resistivity value $\eta_{\perp}=0.05$ used for the reconstruction is close to the value at the location of the magnetic X-point at $\left(x^{\prime} \simeq 9, y^{\prime}=0, z^{\prime}=0\right)$ in Fig. 2, which is about $0.97 \eta_{0}=0.0485$.

Figure 5a shows the field line map from the simulation data, taken in the $x^{\prime}-z^{\prime}$ plane $\left(y^{\prime}=0\right)$, i.e., the field lines in Fig. 2, and then projected onto the reconstruction coor- dinates. The $B_{\mathrm{Z}}$ values in color are the projections of the 3-D magnetic field vectors (at points in the $x^{\prime}-z^{\prime}$ plane) onto the invariant (z-) axis. Although there is only a small angle of $1.3^{\circ}$ between the $y^{\prime}$-axis of the simulation and the $z$ axis of the reconstruction, this angle causes the pronounced anti-symmetric behavior of $B_{\mathrm{z}}$. Figure $5 \mathrm{~b}$ shows the reconstructed map of magnetic field lines (black solid lines), using data from the simulated spacecraft 3, again with the axial field $B_{\mathrm{Z}}$ in color. The horizontal dashed lines denote the spacecraft trajectories (as deduced from the data), projected onto the reconstruction plane. The $x$ and $y$ reconstruction axes are: $\hat{x}=[0.7100,-0.5600,0.4270]$ and $\hat{y}=$ $[0.4300,0.8249,0.3670]$, in the rotated coordinate system. By comparing the reconstructed map with the simulation, we see that the X-point position is well predicted by the resistive MHD reconstruction. Figures 6, 7, 8, and 9 show plots of the predicted values from the reconstruction map based on spacecraft 3 (red dashed line) and those actually measured by the other 5 spacecraft (black line), for the magnetic field, the plasma velocity, the electric field, and the plasma density and pressure, respectively. There is excellent agreement of magnetic field and plasma pressure between the reconstruction and the simulation results themselves, with correlation coefficients $c c=0.999$ and $c c=0.996$, respectively. The plasma density and the electric field components (only $E_{\mathrm{x}}$ and $E_{\mathrm{y}}$ ) 

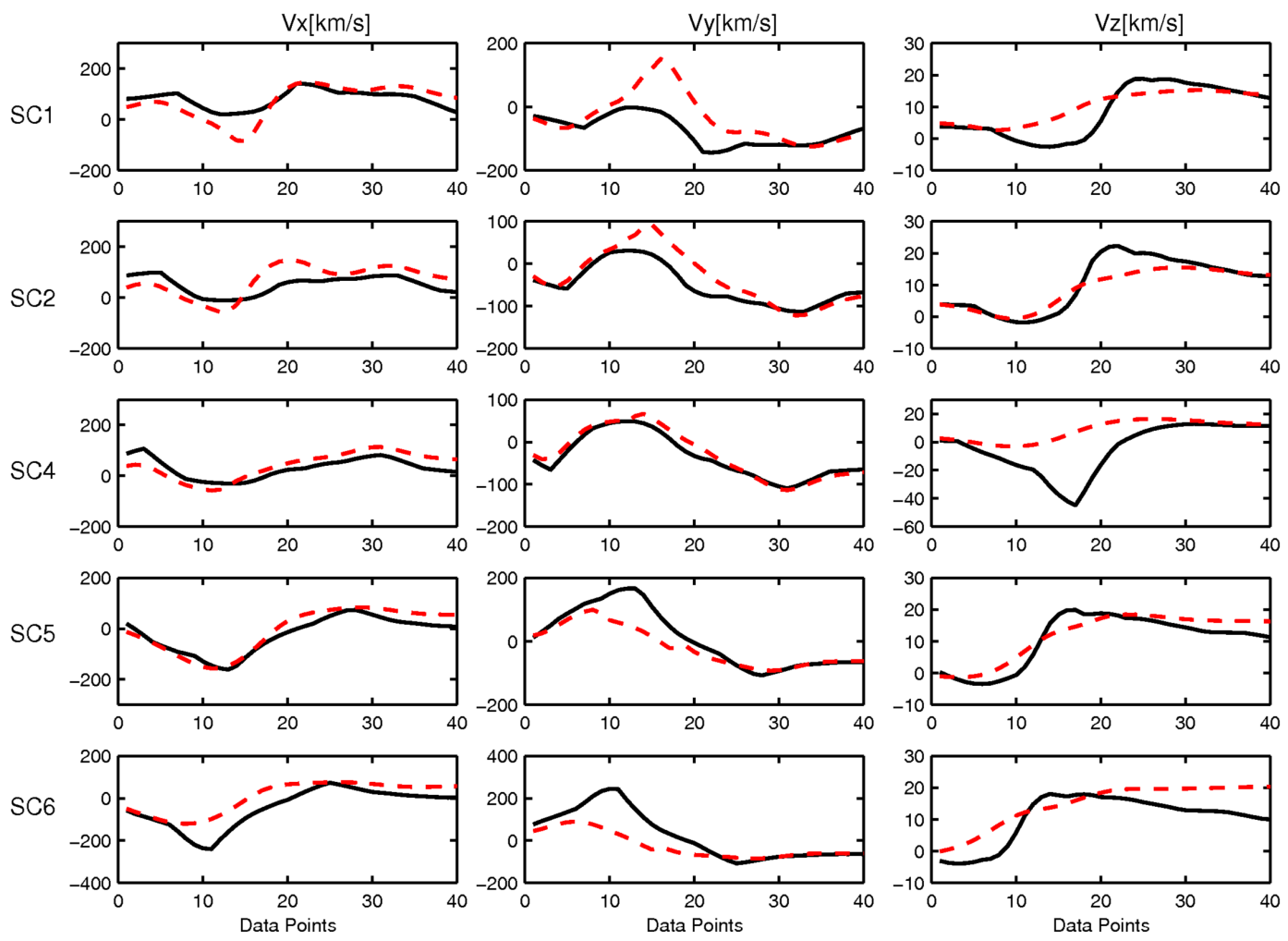

Fig. 7. Snapshot plots of the predicted plasma velocities (red dashed lines) from the reconstruction map based on spacecraft 3 and the corresponding velocities (black lines) taken by the other spacecraft directly from the simulation.

are less well predicted, with $c c=0.961$ and $c c=0.927$, respectively. The plasma velocity is rather poorly predicted, with $c c=0.804$. For spacecraft 4 , which is closest to spacecraft 3 , the plasma velocity is predicted with $c c=0.904$, indicating that the velocity field can be fairly accurately reconstructed, but only within a very narrow region surrounding the path of spacecraft that provides the source data for the reconstruction. To reach these results, we have slightly changed the plasma velocities at $y=0$ so as to obtain a constant axial electric field of $1.353 \mathrm{mV} \mathrm{m}^{-1}$. This step turns out to improve not only the velocity prediction (from $c c=0.284$ to $c c=0.804$ ) but also the other predictions, in particular for density (from $c c=0.914$ to $c c=0.961$ ) and electric field (from $c c=0.747$ to $c c=0.927$ ). The corrections to the velocity at $y=0$ are made by maintaining the velocity component parallel to the field but modifying the one perpendicular to the field so as to produce a constant axial electric field, except as noted in the discussion section. In addition, we experimented with different frame velocities for the reconstruction but did not achieve any further improvement of the velocity prediction.

\section{Summary and discussion}

We have extracted the steady, 2-D resistive MHD reconstruction theory from the more general theory for resistive Hall MHD (Sonnerup and Teh, 2009), by simply neglecting the Hall term and the electron pressure in Ohm's law and momentum equation. The resistive MHD reconstruction algorithm then becomes very simple compared to that needed for resistive Hall MHD. Resistive MHD reconstruction is different from reconstructions based on ideal equations in one important respect: In the presence of resistivity, entropy is no longer conserved along streamlines so that data fitting to produce a curve of entropy $S$ versus stream function $\psi$ is not a part of the integration scheme.

At least two components of the measured electric field, usually those in the spacecraft spin plane, are required for resistive MHD reconstruction to be possible and then only by use of a specified resistivity model. When all three components of the electric field are measured, we show that the values of $\eta_{\perp}$ at points on the spacecraft trajectory can be calculated by use of the y-component of Ohm's law, $\boldsymbol{E}+\boldsymbol{v} \times \boldsymbol{B}=$ $\boldsymbol{\eta} \cdot \boldsymbol{j}$. For simplicity, the resistivity tensor is diagonal and has $\eta_{\mathrm{Z}}=k \eta_{\perp}$, where $k$ is a constant (in our application chosen to be $k=1$ ). We have developed an exact axi-symmetric solution of the steady, 2-D resistive MHD and used it for validation of the resistive MHD reconstruction code. As illustrated 

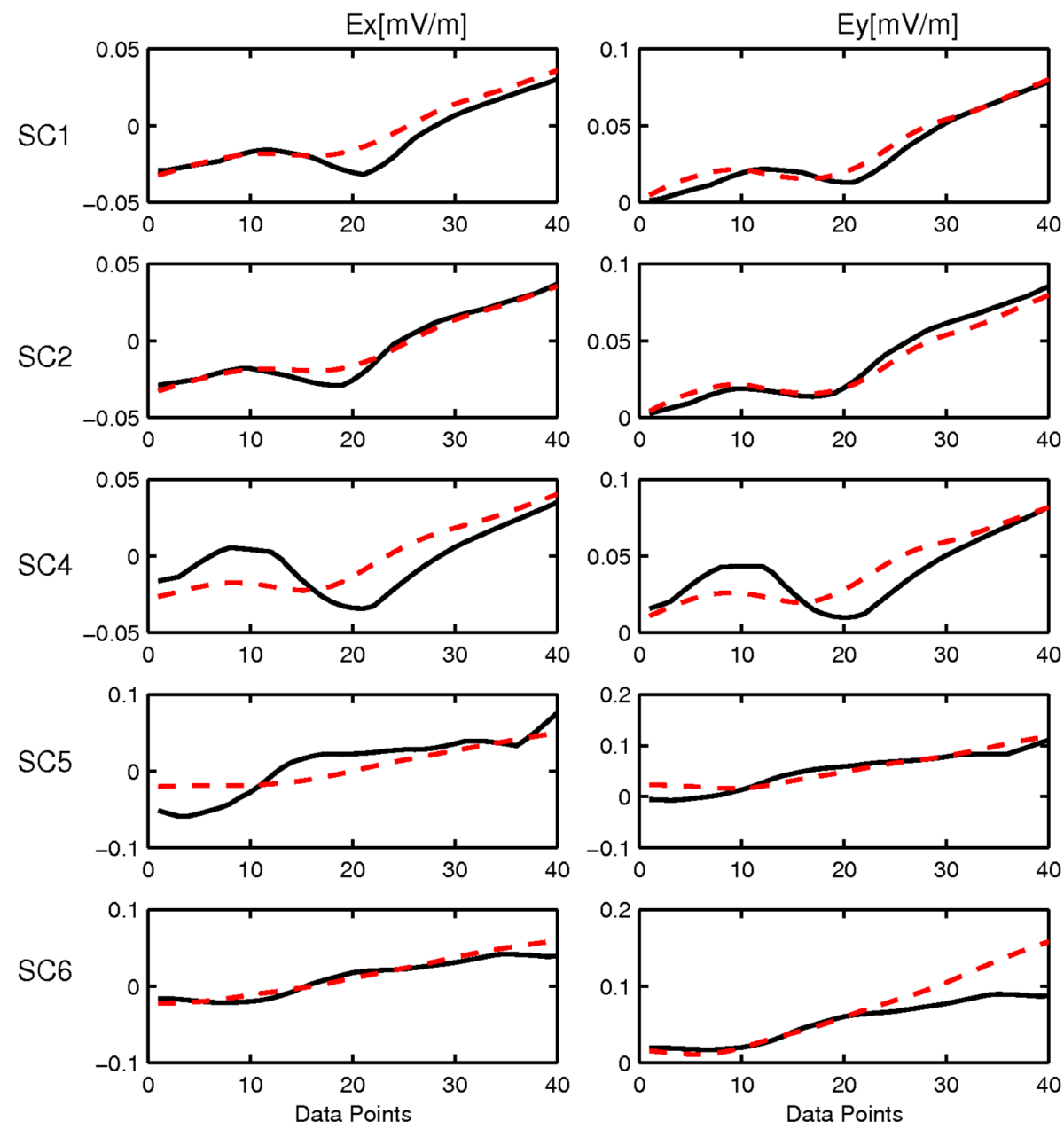

Fig. 8. Snapshot plots of the predicted electric fields (red dashed lines) from the reconstruction map based on spacecraft 3 and the corresponding fields (black lines) taken by the other spacecraft directly from the simulation.

in Fig. 1 and indicated in Table 1, the results show that the field and plasma configurations are accurately reconstructed in an elongated region surrounding the data line at $y=0$, provided the base assumptions of 2-D and time independence are precisely satisfied.

We have then applied the resistive MHD reconstruction to a synthetic reconnection event from a 3-D resistive MHD numerical simulation, in which the resistivity is spatially localized and time independent. Six synthetic spacecraft cross the structure in different places, providing snapshot information. Data from one of the spacecraft is used for the reconstruction and a comparison is then made between the observations by the other five spacecraft and the predictions from the reconstructed field and plasma maps. This was a blind test in the sense that one of us (W. L. T.) did not know the orientation of the rotated coordinate system until after the reconstruction was done. Good agreement is obtained for magnetic field, plasma pressure, and density, with correlation coefficients of $0.999,0.996$, and 0.961 , respectively. However, the elec- tric field and plasma velocity are not predicted with the same high accuracy, the worst prediction being that of the velocity. To improve the velocity prediction, we tried three different schemes: (1) using different frame velocities; (2) modifying the input plasma velocities at $y=0$ to produce a constant axial electric field of $1.353 \mathrm{mV} \mathrm{m}^{-1}$; (3) using the exact invariant axis and frame velocity. Only the second method resulted in significant improvements of the velocity prediction (reaching $c c=0.804$ ). To avoid making the large corrections of the velocity that would be required where the field magnitude is small, the velocity correction is only made where the magnetic field magnitude is greater than some critical value $B_{\mathrm{c}}$. The value $B_{\mathrm{c}}=2 \mathrm{nT}$ was chosen because it gave the best correlation coefficient for the velocity components.

Possible explanations for the lower quality of the predictions for the electric field, plasma velocity and density are: (1) weak time-dependence is present, as illustrated in the measured axial electric field $E_{\mathrm{z} 0}$ (Fig. $4 \mathrm{a}$ ), which is not precisely constant as it should be in a time-stationary 2-D 

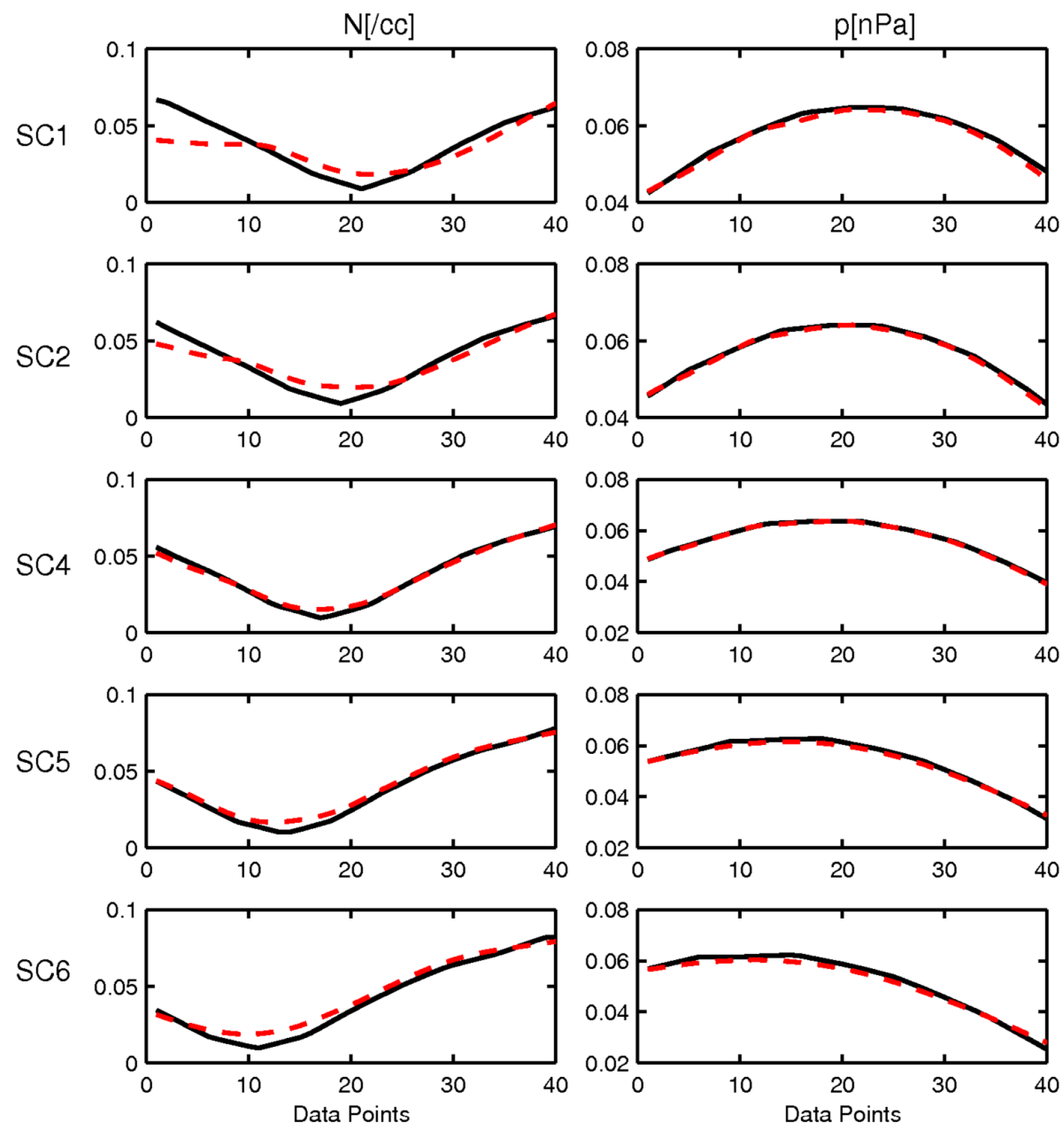

Fig. 9. Snapshot plots of the predicted plasma density $N$ and pressure $p$ (red dashed lines) from the reconstruction map based on spacecraft 3 and the corresponding values (black lines) taken by the other spacecraft directly from the simulation.

structure; (2) the 2-D assumption is also violated to a small extent in the simulation; (3) the resistivity is assumed to be constant everywhere in the reconstruction plane whereas, in the simulation, it has a spatial variation. The combination of these effects could have a significant influence on the behavior of the predicted electric field, plasma velocity, and density. The integration of the vector potential $A$ is performed by use of Eq. (17), in which the value of $\partial^{2} A / \partial y^{2}$ is calculated from Eq. (13). Equation (13) is only weakly coupled to the plasma density and the electric field, which is a possible reason why the magnetic field is obtained with such high accuracy compared to the other quantities. The reason why the plasma pressure is extremely well predicted, but the velocity is not, cannot be readily seen from the equations.

Figure 10 shows the spacecraft-3 based reconstruction map of the magnetic field, resulting from the use of ideal MHD (Sonnerup and Teh, 2008) instead of resistive MHD. Comparison of the maps in Figs. $5 \mathrm{~b}$ and 10 indicates that the resistive MHD reconstruction, with $c c=0.999$, is doing

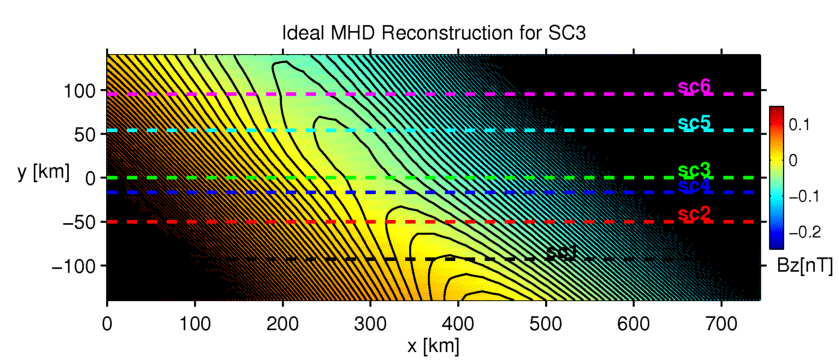

Fig. 10. Ideal MHD reconstruction map of the magnetic field lines (black solid lines) with axial field $B_{\mathrm{Z}}$ in color, based on spacecraft 3 . The format is the same as in Fig. 5.

slightly better than the ideal MHD reconstruction, for which $c c=0.998$. In particular, it is noted that the magnetic X-point can be successfully recovered in the resistive MHD reconstruction while, in ideal MHD reconstruction, it has moved outside the window of view. Therefore the ability we have 
developed in this paper to do resistive MHD reconstruction is of great potential importance for studies of reconnection configurations that include, or are near, the reconnection site itself. However, it must be kept in mind that 2-D reconstructions cannot properly describe 3-D topological features of the field lines.

Although the base assumptions of 2-D and time stationarity for resistive MHD reconstruction are not exactly valid in our simulation model, the reconstruction experiment we have reported on here is a worthwhile first test, showing the viability of the new method. In future studies, the use of various 2-D and 3-D numerical simulation models will be desirable for further diagnosis of the strengths and weaknesses of the method and the conditions under which it gives accurate results. We conclude that resistive reconstruction can become a valuable new tool for the interpretation of space data.

Acknowledgements. This work was supported by NASA grant NNG-05GG26G (B. U. Ö. S.) and NASA grant NNX08AV81G (MMS theory program) (R. E. D.).

Topical Editor R. Nakamura thanks H. Hasegawa and another anonymous referee for their help in evaluating this paper.

\section{References}

Birn, J., Fletcher, L., Hesse, M., and Neukirch, T.: Energy release and transfer in solar flares: Simulation of three-dimensional reconnection, ApJ, 695, 1151-1162, 2009.

Denton, R. E., Sonnerup, B. U. Ö., Birn, J., Teh, W.-L., and Drake, J. F.: Test of method to infer the magnetic reconnection directions from spacecraft data, J. Geophys. Res., 115, A10242, doi:10.1029/2010JA015420, 2010.

Eriksson, S., Hasegawa, H., Teh, W.-L., Sonnerup, B. U. Ö., McFadden, J. P., Glassmeier, K.-H., Le Contel, O., Angelopoulos, V., Cully, C. M., Larson, D. E., Ergun, R. E., Roux, A., and Carlson, C. W.: Magnetic island formation between large-scale flow vortices at an undulating postnoon magnetopause for northward interplanetary magnetic field, J. Geophys. Res., 114, A00C17, doi:10.1029/2008JA013505, 2009.

Hasegawa, H., Sonnerup, B. U. Ö., Dunlop, M. W., Balogh, A., Haaland, S. E., Klecker, B., Paschmann, G., Lavraud, B., Dandouras, I., and Rème, H.: Reconstruction of two-dimensional magnetopause structures from Cluster observations: verification of method, Ann. Geophys., 22, 1251-1266, doi:10.5194/angeo22-1251-2004, 2004.

Hasegawa, H., Sonnerup, B. U. Ö., Klecker, B., Paschmann, G., Dunlop, M. W., and Rème, H.: Optimal reconstruction of magnetopause structures from Cluster data, Ann. Geophys., 23, 973982, doi:10.5194/angeo-23-973-2005, 2005.

Hasegawa, H., Sonnerup, B. U. Ö., Owen, C. J., Klecker, B., Paschmann, G., Balogh, A., and Rème, H.: The structure of flux transfer events recovered from Cluster data, Ann. Geophys., 24, 603-618, doi:10.5194/angeo-24-603-2006, 2006.

Hasegawa, H., Nakamura, R., Fujimoto, M., Sergeev, V. A., Lucek, E. A., Rème, H., and Khotyaintsev, Y.: Reconstruction of a bipolar magnetic signature in an earthward jet in the tail: Flux rope or 3D guide field reconnection?, J. Geophys. Res., 112, A11206, doi:10.1029/2007JA012492, 2007a.
Hasegawa, H., Sonnerup, B. U. Ö., Fujimoto, M., Saito, Y., and Mukai, T.: Recovery of streamlines in the flank lowlatitude boundary layer, J. Geophys. Res., 112, A04213, doi:10.1029/2006JA012101, 2007b.

Hau, L.-N. and Sonnerup, B. U. Ö.: Two-dimensional coherent structures in the magnetopause: Recovery of static equilibria from single-spacecraft data, J. Geophys. Res., 104, 6899-6917, 1999.

Hu, Q. and Sonnerup, B. U. Ö.: Magnetopause transects from two spacecraft: A comparison, Geophys. Res. Lett., 27(10), 14431446, 2000.

$\mathrm{Hu}$, Q. and Sonnerup, B. U. Ö.: Reconstruction of magnetic flux ropes in the solar wind, Geophys. Res. Lett., 28, 467-470, 2001.

Hu, Q. and Sonnerup, B. U. Ö.: Reconstruction of magnetic clouds in the solar wind: Orientations and configurations, J. Geophys. Res., 107(A7), 1142, doi:10.1029/2001JA000293, 2002.

$\mathrm{Hu}, \mathrm{Q}$. and Sonnerup, B. U. Ö.: Reconstruction of two-dimensional structures in the magnetopause: Method improvements, J. Geophys. Res., 108(A1), 1011, doi:10.1029/2002JA009323, 2003.

Lui, A. T. Y., Sibeck, D. G., Phan, T. D., Angelopoulos, V., McFadden, J. M., Carlson, C., Larson, D., Bonnell, J., Glassmeier, K.-H., and Frey, S.: Reconstruction of a magnetic flux rope from THEMIS observations, Geophys. Res. Lett., 35, L17S05, doi:10.1029/2007GL032933, 2008.

Shi, Q. Q., Shen, C., Pu, Z. Y., Dunlop, M. W., Zong, Q.-G., Zhang, H., Xiao, C. J., Liu, Z. X., and Balogh, A.: Dimensional analysis of observed structures using multipoint magnetic field measurements: Application to Cluster, Geophys. Res. Lett., 32, L12105, doi:10.1029/2005GL022454, 2005.

Shi, Q. Q., Shen, C., Dunlop, M. W., Pu, Z. Y., Zong, Q.-G., Liu, Z. X., Lucek, E., and Balogh, A.: Motion of observed structures calculated from multi-point magnetic field measurements: Application to Cluster, Geophys. Res. Lett., 33, L08109, doi:10.1029/2005GL025073, 2006.

Sonnerup, B. U. Ö. and Guo, M.: Magnetopause transects, Geophys. Res. Lett., 23, 3679-3682, 1996.

Sonnerup, B. U. Ö. and Hasegawa, H.: Orientation and motion of two-dimensional structures in a space plasma, J. Geophys. Res., 110, A06208, doi:10.1029/2004JA010853, 2005.

Sonnerup, B. U. Ö. and Teh, W.-L.: Reconstruction of two-dimensional coherent MHD structures in a space plasma: The theory, J. Geophys. Res., 113, A05202, doi:10.1029/2007JA012718, 2008.

Sonnerup, B. U. Ö. and Teh, W.-L.: Reconstruction of two-dimensional coherent structures in ideal and resistive Hall MHD: The theory, J. Geophys. Res., 114, A04206, doi:10.1029/2008JA013897, 2009.

Sonnerup, B. U. Ö., Hasegawa, H., and Paschmann, G.: Anatomy of a flux transfer event seen by Cluster, Geophys. Res. Lett., 31, L11803, doi:10.1029/2004GL020134, 2004.

Sonnerup, B. U. Ö., Hasegawa, H., Teh, W.-L., and Hau, L.-N.: Grad-Shafranov reconstruction: An overview, J. Geophys. Res., 111, A09204, doi:10.1029/2006JA0117, 2006.

Teh, W.-L. and Hau, L.-N.: Evidence for pearl-like magnetic island structures at dawn and dusk side magnetopause, Earth Planets Space, 56, 681-686, 2004.

Teh, W.-L. and Hau, L.-N.: Triple crossings of a string of magnetic islands at duskside magnetopause encountered by AMPTE/IRM satellite on 8 August 1985, J. Geophys. Res., 112, A08207, 
doi:10.1029/2007JA012294, 2007.

Teh, W.-L. and Sonnerup, B. U. Ö.: First results from ideal 2-D MHD reconstruction: magnetopause reconnection event seen by Cluster, Ann. Geophys., 26, 2673-2684, doi:10.5194/angeo-262673-2008, 2008.

Teh, W.-L., Sonnerup, B. U. Ö., and Hau, L.-N.: Grad-Shafranov reconstruction with field-aligned flow: First results, Geophys. Res. Lett., 34, L05109, doi:10.1029/2006GL028802, 2007.
Teh, W.-L., Sonnerup, B. U. Ö., Hu, Q., and Farrugia, C. J.: Reconstruction of a large-scale reconnection exhaust structure in the solar wind, Ann. Geophys., 27, 807-822, doi:10.5194/angeo-27807-2009, 2009. 